\title{
An Approach for Multifaceted Mobile Nodes Sending Information to Manage the Network
}

\author{
C.J. Miao, H.C. Zhou, H.K. Zhang, W. Su \\ National Engineering Laboratory of Next Generation Internet Interconnection Devices \\ Beijing Jiaotong University \\ Beijing, China
}

Abstract-- In traditional host-based mobility management such as MIPv6, mobility node manages the mobility by BU/B A (Binding Update/Binding Acknowledgement). With the development of the mobile network, network-based mobility management such as PMIPv6 is proposed which has been proved more superiority that the host-based mobility solutions. However, As MN knows itself location by convenient. Then it would be more scalable if the mobile nodes can help to manage the network, especially for LMA handover. Besides, it is necessary for the mobile nodes to suggest which network they have access, and how to find a simple way to achieve the scalability and security. For these purposes, we provide a scheme based on mobility node suggestion for PMIPv6 to achieve that the MN takes part in the mobility management.

Keywords- network-based; handover

\section{INTRODUCTION}

Nowadays, the designers of the Mobile Internet try their best to improve the performance of the network such as scalability and security. By the end of June 2014, the number of the mobile phone users has been increase to 632 million [1]. As the rapid growing number of the mobile nodes (MNs), there seems to be a lot of problems that must be faced to, especially such as how to deal with the users in different ways and how can the user tell the Internet Service Provider or the routers what kind of the server the user would like.

The recent study have been pointed out that the current trend has been focused mostly on realizing all-IP mobile networks, as they are expected to connect the Internet and telecommun ication networks tightly.

Based on the different requirement for the terminal in the literature [2-10], there are three different ways for the mobile nodes to access the Internet: [11]

Firstly, MNs take part in the mobility management significantly, such as MIPv4 [2], MIPv6 [3], NEMO [4], MCoA [5], HMIP [6]. In these protocols, MNs are the senders or receivers of the BU/BA (Binding Updates/ Binding Acknowledges), with which the user can express its requirements for the network conveniently.

Secondly, MNs does not take part in the mobility management, such as PMIPv6 [7] and some extended protocols [8-11]. In these protocols, when a mobile node enters a PMIP domain and attaches to an access network, the MAG (Mobility Access Gateway) on the access link detects the attachment of the $\mathrm{MN}$ and completes the binding registration with the MN's local mobility anchor. The MAG manages the mobility instead of the MN in MIPv6. So it is really difficult for a $\mathrm{MN}$ express its requirements.

Thirdly, MNs take part in the mobility management by accident such as in the literature [12]. It provides taxonomy of the most common scenarios that require direct interaction between MIPv6 and PMIPv6.

PMIPv6, as a representative of the network-based mobile protocol, is proved to have the superiorities. Unlike host-based mobility management, network-based mobility management does not require any modification of MNs. The requirement for modification of MNs can be considered one of the primary reasons MIPv6 has not been widely deployed in practice, although several commendable MIPv6 enhancements have been reported over the past years. Therefore, no requirement for modification of MNs is expected to accelerate the practical deployment of PMIPv6. It is expected that network-based mobility management would enhance manageability and flexibility by enabling network service providers to control network traffic and provide differentiated services and so on. The wire less users can connect to the Internet with the MAGs. In the network, MN, as the edge equipment, is the receiver of mobility service.

After several decades of the fast development, the customers should be more significant [13]. Network-based mobility management faces to many problems. With the crowed of the Internet, how does the network avoid providing a worse service for the important user but a better for the unimportant? When a MN move from a domain to another, how can the new LMA find the previous LMAs? How to improve the scalability and security for the MN? It is not a simple way for the network-based mobility management knows what the MN wants and where the MN locates. However, MN is the most understanding what itself wants and should have the right for the network management for it to light the load of the network and let the network know where it is.

We outline the network model and the topology, describe the approach of $\mathrm{MN}$ sending information to manage the network (MNSIMN), focus on the function of MN, and analyze its benefits and disadvantages.

\section{NETWORK MODEL}

In the network, $\mathrm{MN}$, as the edge equip ment, is the receiver of mobility service, so it should have its right to choose the service. 
Network domains comprise the LMA (Local Mobility Anchor) and are used for providing mobility service for the mobile nodes. Access networks connect with PMIPv6 domains through MAGs located at edges of either PMIPv6 domains or Access networks. Similar to PMIPv6 [7], we consider a network in which access networks are separated from PMIPv6 domain, as illustrated fig. 1. Access networks are composed of variety of the MNs. Two Network domains connect through routers located at edges of either network domains or Access networks.

The MNs are assumed to support IPv6; Each MAG knows all the LMA's address and MAG can choose a suitable LMA. For facilitate the description, we assumed the terminal was $\mathrm{MN1}$, and the steps of MN1 moved as follows:

1) MN1 accessed MAG1 to attach the Network Domain, binding to LMA1;

2) MN1 moved in the same Network Do main from MAG1 to MAG2, still binding to LMA 1;

3) MN1 moved to MAG3 in the PMIPv6 Domain, then accessed MAG3, but in the cache of the MAG3, seems to be binding to LMA2;

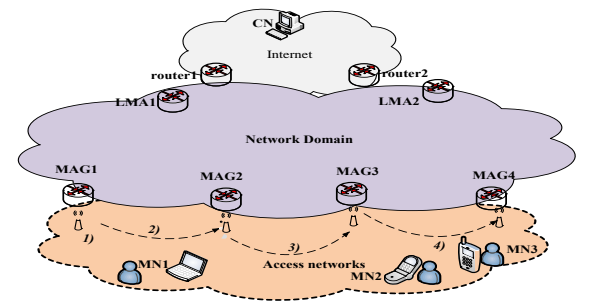

FIGURE I. ILLUSTRATION NETWORK MODEL.

\section{ANAPPROACH OF MN SENDING INFORMATION TO MANA GE THE NETWORK}

Due to its salient feature in offering the $\mathrm{MN}$ some rights to send the information to the network, we call the proposed approach MNSIMN. We assume that MN knows the last LMA address and the home network prefix(es) that the last LMA provide. The first time for $\mathrm{MN}$ to access MAG is similar to PMIPv6. In our description, the MN1 access the MAG1 is the first time to access the network.

Since the process 4) is the same to the process 2) in in the fig 1 . We convert the fig. 1 to fig. 2 describe the movement in the same domain.

a) When the MN1 attaches to the access link, it will send a Router Solicitation (RS) message, which contains the identifications of MN1;

b) MAG1 authenticates identification of MN1;

c) MA G1 sends PBU, which is similar to the PMIPv6;

d) MAG1 receives $\mathrm{PBA}$, which is the similar to the PMIPv6;

e) The MAG1 on the access link will respond to the Router Solicitation message with a Router Advertisement (RA) message. The RA message will carry the MN1's home network prefix (es), default-router address, LMA1's address, domain number and other address configuration parameters. MN1 configuration its address according to the prefix (es) and its own identification by DHCP or other which is descripted in the references [7]. MN1 must record the LMA1 address, domains number and its own address in cache;

f) When MN1 moved from MAG1 to MAG2, MN1 send RA, which contains not only the identifications, but also the MN1 address and LMA1 address;

g) MAG2 authenticates identification of MN1, and finds if the LMA1 is in the same Network Domain with MAG2. If in the MAG2 the LMA1 is a suitable LMA, it will send PBU/PBA just as usual, go to c. Otherwise, such as MAG3 is shows in fig. 3 , go to the step h);

h) MAG3 send PBU to LMA2, with the prefix (es) and the LMA 1 address;

i) LMA2 receive the $\mathrm{PBU}$, according to the LMA1 address then send to LMA1 a message, we named it LTL (LMA To LMA) message with the PBU that the MAG3 send to LMA2. If the LMA1 receive LTL from LMA2, it must delete the BC;

j) LMA 1 reply LMA2 a LTL Acknowledge message.

k) LMA2 add the prefix (es) and MN identifications, to build an BCE for MN.

1) LMA2 reply a PBA for MAG3; and then MAG3 rebuilds a BULE for MN.

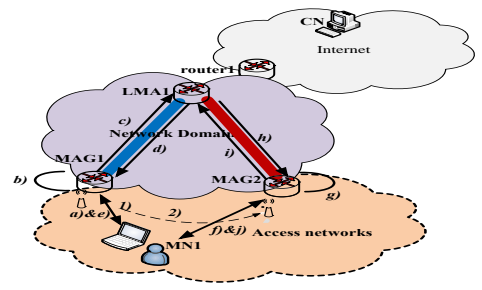

FIGURE II. MN1 ACCESS THE PMIPV6 DOMAIN1.

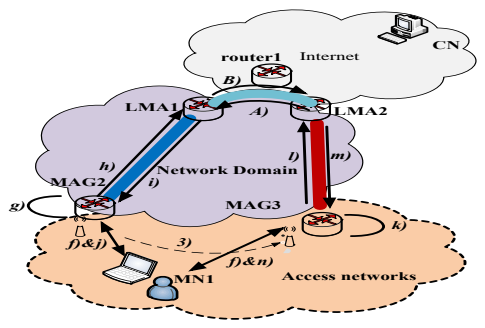

FIGURE III. MN1 move access MAG33.

MAG3 sends RA with LMA2 address and the same prefix (es) and the Network Domain number to MN1. MN1 modifies the record in the cache.

\section{EVALUATION OF THE APPROACH}

\section{A. Scalability and Supportion of LMA Handover and Multihome Functions}

MNSIMN is a system with excellent scalability. No one knows the location of $\mathrm{MN}$ in the history better than itself. The system lets $\mathrm{MN}$ have its own function to participate the 
location management and the movement management, and with the suggestion of the MN, MAG can easily find the LMA if MAG does not know which LMA to connect. MN can choose the LMA may bring the customers an advanced service experience. The new function is more humanist and suitable for feeling. In steps 3) (MN1 moved to MAG3 in the PMIPv6 Domain), the systems provide an extension function of LMA handover.

\section{B. Handover Faster}

Handover is with a lower latency than MIPv6 and PMIPv6, as is shown below.

Firstly, in the steps of 1), all the latency except the step e) is the same to PMIPv6. $T_{P B U-M}$ and $T_{P B A-M}$ means the PBU and PBA signaling latency in MNSIMN, $T_{P B U-P}$ and $T_{P B A-P}$ means PBU and PBA signaling latency in PMIPv6.

$$
T_{P B U M}=T_{P B U P} ; T_{P B A-M}=T_{P B A P}
$$

In the step f) and e), MN send it suggestion with RS, and receive the message of the LMA address and PMIPv6 domain number with RA. This system does not bring more signaling between the MN and MAG. Only increase the bites of the RS and RA. The increasing of such a little bites much smaller than the latencys of the sending or receiving the RS or RA. $T_{R S-M}$ and $T_{R A-M}$ means the time of the RA and RA in the MNSIMN system, $T_{R S-P}$ and $T_{R A-P}$ means the times that in PMIPv6. Based on the analysis above, we can get:

$$
T_{R S-M} \approx T_{R S-P} ; T_{R A-M} \approx T_{R A-P}
$$

Secondly, in the process $\mathrm{g}$ ), it should be save the time that the MAG latency searching the LMA at the steps 2), in this section, MAG does not know which LMA should MN bind, in PMIPv6, the MAG must request from AAA [14], but in this system, MN1 tells MAG2 which LMA it connect before, and then MAG2 send the PBU and receive PBA immediately. It can save lot of the tedious signaling in the network. $T_{2 M A G-M}$ and $T_{2 L M A-M}$ mean the latency of the MAG process the step g) and LMA process the MNSIMN. $T_{2 M A G-P}, T_{A A A R-P}, T_{A A A A-P}, T_{2 L M A-P}$ mean in PMIPv6 M N register a new MA G's signal latency.

Thirdly, in process i) and j), in the steps of 3), the MAG thinks it should be change the LMA1 to LMA2. It instructs that the network still effect in the mobility management, so $\mathrm{MN}$ is only a secondary in the network management.

$$
T_{3 L T L R} \text { and } T_{3 L T L A} \text { mean the latency of i) and j) in step 3) in }
$$
MNSIMN. $T_{L M A 3-L M A 2}+T_{L M A 2-L M A 3}+T_{L M A-A A A A}+T_{L M A-A A A R}$ means the latency of LMA redirect in PMIPv 6.
With the suggestion of the MN, it can save a lot of latency in the step 2) and 3); $T_{2-M}$ and $T_{3-M}$ mean in step 2) and 3) the signaling latency of the network in MNSIMN. $T_{2-P}$ and $T_{3-P}$ mean in step 2) and 3) the signaling latency of the network in PMIPv6. Obviously, we can get the formula as follows:

$$
\begin{aligned}
& T_{2 M}=T_{R A M}+T_{R S M}+T_{P B U M}+T_{P B U M}+T_{2 M A G M}+T_{2 L M A M} \\
& T_{2 P}=T_{R A P}+T_{R S P}+T_{P B U P}+T_{P B A P}+T_{2 M A G P}+T_{A A A R P}+T_{A A A A P}+T_{2 L M A P} \\
& T_{3 M}=T_{R A M}+T_{R S M}+T_{P B U M}+T_{P B A M}+T_{3 M A G M}+T_{3 L M A M}+T_{3 L T L R}+T_{3 L T L A} \\
& T_{3 P}=T_{R A P}+T_{R S P}+T_{P B U P}+T_{P B A P}+T_{3 M A G P}+T_{A A A R P}+T_{A A A A P}+T_{3 L M A P}+T_{L M A 3 ~ L M A 2} \\
& +T_{L M A 2 L M A 3}+T_{L M A A A A A}+T_{L M A A A A R} \\
& \square 2=T_{2 P} \quad T_{2 M}=T_{A A A R P}+T_{A A A A P}>0 \\
& \square 3=T_{3 P} \quad T_{3 M}=T_{A A A R P}+T_{A A A A P}+T_{L M A A A A A}+T_{L M A A A A R}>0
\end{aligned}
$$

The latency of signaling is obviously shown at the formula above, our system can reduce the latency in the signal.

\section{C.Real Implementation of MNSIMN}

The MNSIMN scheme has been implemented in a real testbed in our laboratory. We use the topology showed in Fig. 2. MN performs handover between MAG1 and MAG2 50 times. The handover latency is shown in Fig. 4.

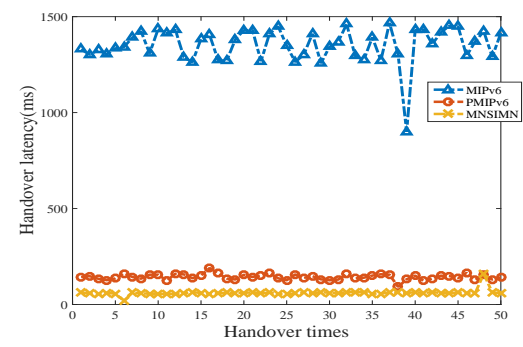

FIGURE IV. THE HANDOVER LATENCY.

The average handover latency of MIPv6 is $1248.2 \mathrm{~ms}$, the latency of PMIPv 6 is $121.32 \mathrm{~ms}$, and the latency of MNSIMN is $53.12 \mathrm{~ms}$. The result shows that MNSIMN scheme can reduce the handover latency of the network.

\section{CONCLUSIONS}

This article aims to provide an approach of the $\mathrm{MN}$ sending some information to manage the network. It is a method in achievement the scalability and support for LMA handover and multifaceted $\mathrm{MN}$ in the network. It is proved to be faster handover than the MIPv6 and PMIPv6. Similar to this idea, such of the system can also be extending to the handover in two different kinds of network domains. As MN can send the information to the network, the costumers of the Internet can play an important part in the lightweight management to cut down the load of the network, in order to better fulfill the function of the mobility. 


\section{ACKNOW LEDGEMENTS}

Supported by the 973 Program, China (No.2013CB329100); Beijing Higher Education Young Elite Teacher Project (No.YETP0534); China Internet Network Information Center, DNSLA B.

\section{REFERENCES}

[1] 34th Chinese Internet development report, http://www.cnnic.net

[2] C. Perkins, P. Calhoun, "Mobile IPv4 Challenge/Response Extensions (Revised)," IETF RFC 4721, January 2007

[3] C. Perkins. D. Johnson, and J. Arkko, "Mobility Support in IPv6," IETF RFC 6275, July 2011.

[4] V. Devarapalli, R. Wakikawa, A. Petrescu, P. Thubert, "Network Mobility (NEMO) Basic Support Protocol," IETF RFC 3963, January 2005.

[5] R. Wakikawa, "Flow Binding in Mobile IPv6 and Network Mobility (NEMO) Basic Support,” IETF RFC 6089, January 2011.

[6] H. Soliman, C. Castelluccia, K. E. Malki, L. Bellier, "Hierarchical mobile IPv6 mobility management (HMIPv6), " IETF RFC 5380, Oct ober 2008.

[7] S. Gundavelli, "Reserved IPv6 Identifier for Proxy Mobile IPv6," IETF RFC 6543, May 2012.

[8] S. Gundavelli, "Extensions to Proxy Mobile IPv6 Motivation, "IETF Draft, draft-gundavelli-net ext-extensions-motivation-00, May 05, 2009

[9] B. Sarikaya, F. Xia, "PMIPv6 Multihoming Support for Flow Mobility, 'IETF draft, draft-sarikaya-netext-fb-support-extensions-00, February 1, 2010.

[10] T. Tran, Y. Hong, "Virtual interface for supporting multihoming and inter technology handover," IETF draft, draft-trung-netext-virtualinterface-01, Oct ober 27, 2009.

[11] Akyildiz, I. F., McNair, J., Ho, J. S. M., Uzunalioglu, H., Wang, W. (1999). "Mobility management in next-generation wireless systems," Proceedings of the IEEE, 87(8), 1347-1384.

[12] G. Giaretta (Qualcomm ), "Interactions Between PMIPv6 and MIPv6: Scenarios and Related Issues," IETF RFC 6612, May, 2012

[13] D. Clark, M. Blumenthal, "The end-to-end argument and application design: the role of trust," in: Proceedings of TPRC, August 2007.

[14] C. de Laat, G. Gross, L. Gommans, et al., Generic AAA Architecture, IET F RFC 2903, August 2000. 